\title{
Anomalous impurity effect on magnetization in frustrated one-dimensional ferro- and ferrimagnets
}

\author{
Masanori Kohno and Xiao $\mathrm{Hu}$ \\ Computational Materials Science Center, National Institute for Materials Science, Tsukuba 305-0047, Japan
}

(Dated: May 20, 2018)

\begin{abstract}
Significant decrease of spontaneous magnetization in frustrated one-dimensional ferro- and ferrimagnets due to non-magnetic impurities is predicted. Using the density-matrix renormalization group method and the exact diagonalization method, we confirm that the total spin can vanish due to a single impurity in finite chains. Introducing the picture of magnetic domain inversion, we numerically investigate the impurity-density dependence of magnetization. In particular, we show that even with an infinitesimal density of impurities the magnetization in the ground state is reduced by about $40 \%$ from that of the corresponding pure system. Conditions for the materials which may show this anomalous impurity effect are formulated.
\end{abstract}

PACS numbers: $75.10 . \mathrm{Pq}, 75.10 . \mathrm{Nr}, 75.30 . \mathrm{Cr}, 75.30 . \mathrm{Hx}, 75.60 . \mathrm{Ch}$

Frustrations in quantum spin systems have attracted much attention for its potential to exhibit new phenomena which have never been observed in unfrustrated systems. Various possibilities in frustrated systems have been suggested, such as exotic excitations near critical points [1], incommensurate orders in magnetic fields [2], chiral orderings [3, 4, 5, 6] and disordered ground states $[7,[8]$. The property we will discuss in this paper is also one of the phenomena where frustrations play an essential role, and will never be observed in unfrustrated systems. The property is an impurity effect on frustrated one-dimensional ferro- and ferrimagnets.

Usually, a small amount of impurities has little influence on bulk magnetic quantities, since the mean distance between impurities is so long that the correlation between them is very weak and usually they affect only local quantities. However, in some special situations, a small amount of impurities can cause a bulk effect. An example is the impurity-induced antiferromagnetic longrange order (AFLRO) in quasi-one-dimensional spin$1 / 2$ spin-gap systems, which was thoroughly investigated by theoretical [9, 10, 11], numerical [12, 13] and experimental 14, 15] approaches. The main feature of this effect is roughly explained as follows: Without an impurity, spins form dimers locally. By introducing nonmagnetic impurities, moments are induced around impurity sites. The moments couple one to another and exhibit antiferromagnetic alignment. Hence, AFLRO and low-energy spin-wave excitation appear in the background of high-energy triplet excitation. For this effect, the spin gap and the correlations between induced moments play an essential role. In this paper, we present another example that a bulk quantity, magnetization, is substantially influenced by a small amount of impurities due to a different mechanism from that of the impurityinduced AFLRO. The impurity effect will be realized even with an infinitesimal density of impurities in frustrated ferro- and ferrimagnetic chains that satisfy the conditions we will present in this paper. Thus, in such systems the magnetization in the ground state will be significantly reduced from that of the corresponding pure
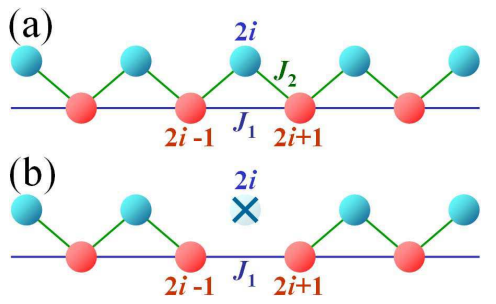

FIG. 1: Lattice structure of models 1 and 2 (a) without an impurity and (b) with an impurity at site $2 i$.

systems even with a usually-negligible amount of impurities. We notice that without the knowledge developed in this work, reduction of magnetization from the expected values tends to be explained by assuming complex, higher order interactions such as the Dzyaloshinsky-Moriya interaction or interchain antiferromagnetic couplings.

In order to demonstrate the anomalous effect of nonmagnetic impurities on frustrated ferro- and ferrimagnets, we consider the minimal models defined by the following Hamiltonian:

$$
\mathcal{H}=J_{1} \sum_{i} \boldsymbol{S}_{2 i-1} \cdot \boldsymbol{S}_{2 i+1}+J_{2} \sum_{i} \boldsymbol{S}_{i} \cdot \boldsymbol{S}_{i+1}
$$

where $\boldsymbol{S}_{i}$ denotes the spin operator at site $i$. The lattice structure is shown in Fig. 1 (a). In model 1, the spin lengths of all spins are one half. The coupling constant $J_{1}$ is antiferromagnetic $\left(J_{1}>0\right)$, and $J_{2}$ is ferromagnetic $\left(J_{2}<0\right)$. In model 2 , the spin lengths at even sites are one half, and those at odd sites are one; both coupling constants are antiferromagnetic $\left(J_{1}\right.$ and $\left.J_{2}>0\right)$. Model 1 is nothing but the one proposed by Hamada and his coworkers in Ref. [16]. Model 2 can be reduced to wellknown models by neglecting $J_{1}$ or $J_{2}$ : At $J_{2}=0$ this model is equivalent to the $S=1$ Heisenberg chain and free spins, and at $J_{1}=0$ it is nothing but the spin-alternating Heisenberg chain. The ground states of models 1 and 2 become ferro- and ferrimagnetic, respectively, when $\left|J_{2}\right|$ is sufficiently larger than $J_{1}$. Hereafter, the number of unit cells and the number of sites are denoted by $L$ and $N_{s}$, 


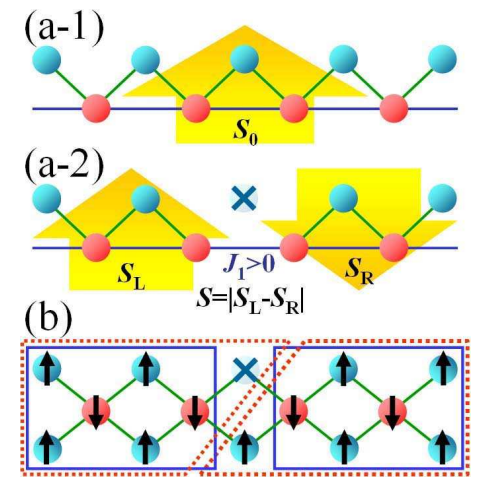

FIG. 2: (a) Schematic picture of magnetic domains (a-1) without an impurity and (a-2) with an impurity. (b) Ferrimagnetic chain on a bipartite lattice, where the spin lengths of all spins are one half, and all coupling constants are antiferromagnetic.

respectively, and open boundary conditions are applied. In models 1 and $2, L=N_{s} / 2$.

When a non-magnetic impurity is doped at an odd site, the system is decomposed into two pure systems. Then, the situation is rather trivial. Thus, we first concentrate on the case where an impurity occupies an even site as shown in Fig. 10 (b). Since the interactions from the impurity site are removed, the remaining interaction between the spins adjacent to the impurity site is $J_{1}$, which is antiferromagnetic. Hence, we expect that the total spin $S$ in the ground state, which corresponds to the spontaneous magnetization, becomes $\left|S_{L}-S_{R}\right|$, where $S_{L}$ and $S_{R}$ are those of the blocks to the left and right of the impurity site, respectively. The expected picture of this inversion of magnetic domains is schematically shown in Fig. 2 (a). As a special case where $S_{L}=S_{R}$, the total spin will vanish due to a single impurity.

In order to confirm this substantial decrease in spontaneous magnetization, we calculated $S$ in the ground states of models 1 and 2 with a single impurity put at all possible even sites in up to 40-site chains with even $L$ by the density-matrix renormalization group (DMRG) method 17 and the exact diagonalization method. The coupling constants are set to be $J_{1}=0.1$ and $\left|J_{2}\right|=1$. We calculated the total spin $S$ in the ground state by using the formula $S(S+1)=\left\langle\left(\sum_{i} \boldsymbol{S}_{i}\right)^{2}\right\rangle\left(=\left\langle\sum_{i, j} \boldsymbol{S}_{i} \cdot \boldsymbol{S}_{j}\right\rangle\right)$, where $i$ and $j$ run over all sites, and \langle\rangle denotes the expectation value in the ground state. The numerical results on the total spin $S$ satisfied the relation $S=\left|S_{L}-S_{R}\right|$ in all the cases we have investigated. There is no mathematical proof on this relation for quantum spin systems with frustrations, hence it is nontrivial. The physical picture of the domain inversion can be intuitively understood by considering the corresponding Ising models, for which this relation holds with total spin $S$ replaced with total $z$-component of spins $S^{z}$.

It should be noted, on the other hand, that in unfrustrated ferrimagnetic chains on bipartite lattices such as shown in Fig. 2 (b), this anomalous impurity effect does not occur, since the sign of the effective coupling between

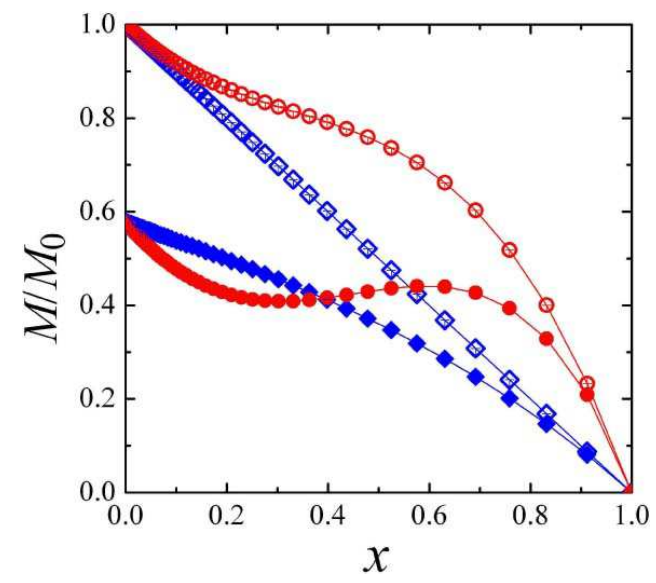

FIG. 3: Impurity-density dependence of magnetization. The magnetizations without an impurity are denoted by $M_{0}$. Solid diamonds and circles denote the results on models 1 and 2, respectively. Open symbols denote those of the corresponding unfrustrated systems by setting $J_{1}$ to be ferromagnetic.

domains does not change due to impurities: In both definitions of domains denoted by solid and dotted lines in Fig. 2] (b), the sign of the effective coupling remains the same before or after impurity doping. Actually, in these systems, ferrimagnetic ground states are ensured by the Marshall-Lieb-Mattis theorem 18, 19 with or without an impurity.

Based on the above numerical results for models 1 and 2 doped with an impurity, it is natural to expect that the total spin $S$ is expressed in terms of those in domains $\left(S_{k}\right)$ as

$$
S=\left|\sum_{k}(-1)^{k} S_{k}\right|,
$$

when impurities are doped at even sites. Taking this relation into account, we have calculated magnetization $M$ in an infinitesimal magnetic field with impurities randomly distributed on a chain, where impurities can sit not only on even sites but also on odd sites. Here, the magnetization $M$ in an infinitesimal magnetic field is expressed in terms of the total spin $S_{l}$ in the $l$-th isolated cluster as $M=\sum_{l} S_{l}$. To be concrete, we have calculated the average of $M$ over 10,000 chains. Each chain has $100 / x$ sites and 100 randomly distributed impurities, where $x$ is the impurity density. The numerical result on the impurity-density dependence of magnetization is shown in Fig. 3] Magnetizations of models 1 and 2 are drastically reduced due to impurities (solid diamonds and circles, respectively). In particular, in the limit of small impurity-density, the magnetizations decrease down to about $57.7 \%$ of those of the corresponding pure systems; $M(x \rightarrow 0) \simeq 0.577 \times M(x=0)$.

This feature is contrasted with that without frustrations: As an example, we consider the model 1 with all coupling constants ferromagnetic. In this model, the ground state is ferromagnetic with or without an impu- 


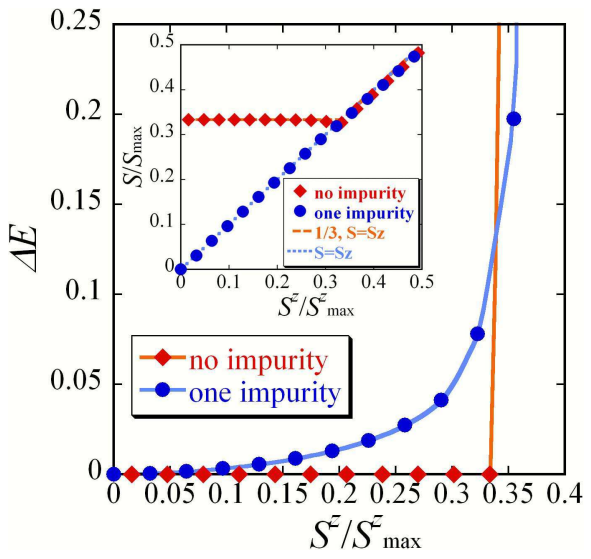

FIG. 4: Ground-state energies as a function of $S^{z}$ measured from that of $S^{z}=0$ in a 42 -site cluster of model 2 with $J_{1}=0.1$ and $J_{2}=1$ without an impurity (diamonds) and with an impurity at the 20-th site (circles). Solid lines are guide to eyes. The inset shows the calculated $S$. Dashed and dotted lines indicate typical behaviors of a ferrimagnet and a disordered state, respectively. The $S$ and $S^{z}$ of the fully polarized state are denoted by $S_{\max }$ and $S_{\max }^{z}$, respectively.

rity. Thus, the magnetization decreases by the amount of the spins at impurity sites. Namely, the magnetization linearly decreases as a function of the impurity density, i.e. $M / M_{0}=1-x$, as shown in Fig. 3 (open diamonds). Since the corresponding Ising models show the same impurity effect for the $z$-component of spins, it is expected that the effect shown here will also be realized in XXZ models with Ising-like anisotropy.

Now, let us consider excitations in doped systems with non-magnetic impurities. We calculated energies of a $42-$ site chain of model 2 with open boundary conditions, where an impurity is put at the 20-th site. The coupling constants are set to be $J_{1}=0.1$ and $J_{2}=1$. The finite-size algorithm of the DMRG method [17] is applied with truncation number up to $m=150$. We performed 10 sweeps and confirmed convergence by calculating $S(S+1)=\left\langle\sum_{i, j} \boldsymbol{S}_{i} \cdot \boldsymbol{S}_{j}\right\rangle$. In the inset of Fig. 目 we plot the calculated $S$ with respect to total $z$-component of spins $S^{z}\left(=\sum_{i} S_{i}^{z}\right)$. The calculated values of $S$ almost coincide with the typical behaviors of a ferrimagnet and a disordered state (dashed and dotted lines, respectively), indicating that the wavefunctions with various $S^{z}$ 's are well converged. This figure also shows that the ground state changes from a ferrimagnetic state to a spin-singlet state due to a single impurity. The ground-state energies within the subspaces of fixed $S^{z}$ measured from that of $S^{z}=0$ are shown in Fig. 4 This figure suggests that there is a low-energy continuous excitation from a spinsinglet state 20]. This feature is contrasted with that of the Ising model where the lowest excitation has a finite gap of the order of $J_{1}$ or $J_{2}$ independently of the cluster size. The ground states with small $S^{z}$ 's in doped quantum systems are almost degenerate, which would be reflecting ferromagnetic fluctuations in domains.

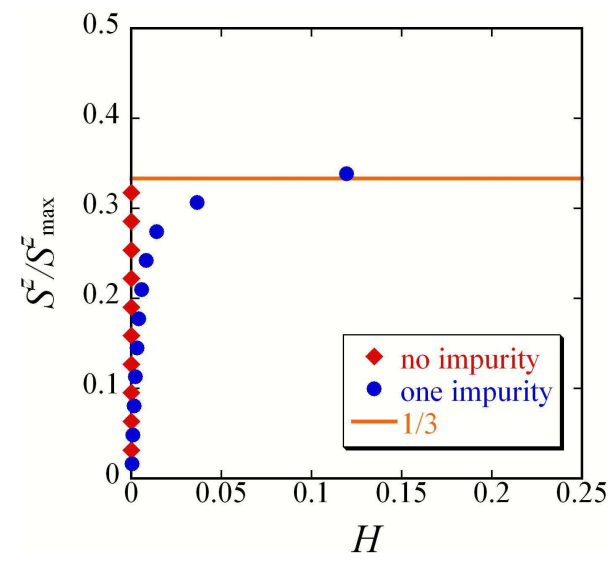

FIG. 5: Magnetization curve of model 2. The parameters are the same as those in Fig. 4

We calculated magnetic field $H$ by using a discretized form of the derivative of energy $E$ with respect to $S^{z}: H=$ $\partial E / \partial S^{z} \simeq\left\{E\left(S_{n+1}^{z}\right)-E\left(S_{n}^{z}\right)\right\} /\left\{S_{n+1}^{z}-S_{n}^{z}\right\}$, where $S_{n}^{z}=n$ or $n+0.5$ with or without an impurity. $(n=0,1, \cdots)$ The result on the magnetization curve is shown in Fig. 5. The magnetic field required for the magnetization to recover up to the spontaneous magnetization of the pure system is about 0.1 which is the order of $J_{1}$ as expected from the picture of domain inversion (Fig. 2 (a)).

Based on the above considerations, we list the conditions for the anomalous impurity effect:

1. The system should be one-dimensional. Namely, interactions between chains should be much smaller than those in chains.

2. The ground state without an impurity should have spontaneous magnetization.

3. Local interactions near impurity sites should be set such that the effective interaction between magnetic domains changes from ferromagnetic to antiferromagnetic due to impurities.

The third condition leads to frustration.

The models that satisfy the above conditions will exhibit the anomalous impurity effect. For example, the decorated triangle chains (Figs. 6] (a-1) and (a-2)) and the diamond-like chain (Fig. [6 (b)) will be the models that exhibit this effect with all spins one half and all coupling constants antiferromagnetic. In the decorated triangle chains, when $J_{2}$ is sufficiently larger than $J_{1}$, the spins on decorating sites align parallel, resulting in a ferrimagnetic ground state. If an impurity is doped on the top site of a triangle, the remaining interaction between the spins adjacent to the impurity is $J_{1}$, which is antiferromagnetic. Thus, the domain inversion and substantial decrease in magnetization due to impurities are expected. Actually, we have confirmed by exact diagonalization that the total spin $S$ in the ground state behaves as $S=\left|S_{L}-S_{R}\right|$, when an impurity is doped on top sites 


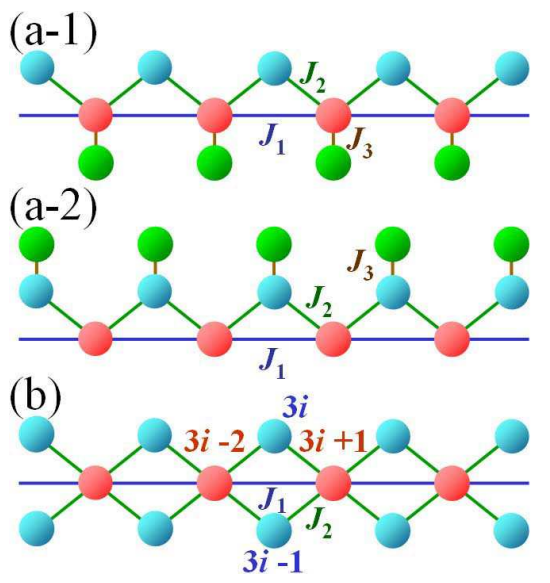

FIG. 6: Possible models for the anomalous impurity effect with spins one half and coupling constants antiferromagnetic. (a) Decorated triangle chains. (b) Diamond-like chain.

of triangles in up to 24-site clusters with $J_{1}=0.1, J_{2}=1.0$ and $J_{3}=0.521$.

In the case of the model in Fig. 6 (b), the ground state becomes ferrimagnetic, when $J_{2}$ is sufficiently larger than $J_{1}$. If an impurity is doped at site $3 i$, the effective interaction between the spins at sites $3 i-2$ and $3 i+1$ is mainly determined by the three-site Hamiltonian of sites $3 i-2$, $3 i-1$ and $3 i+1$. In order for the effective interaction to be antiferromagnetic, $J_{1}$ has to be larger than the effective coupling by $J_{2}$ 's through the spin at site $3 i-1$. If such a parameter can be chosen, the magnetic domain inversion due to impurities will be realized. In this paper, we do not intend to determine the precise boundaries for this effect, since in delicate systems such as that of Fig. [6 (b) the phase boundary will depend on the system size. Instead, we would like to emphasize that, as demonstrated in this paper, there actually exist systems that exhibit this impurity effect in some parameter regimes for frustrated ferro- and ferrimagnets in one dimension.

In summary, we have investigated effects of nonmagnetic impurities on frustrated ferro- and ferrimagnets in one dimension by the DMRG method and the exact diagonalization method. Based on the numerical results, we pointed out that in these systems a small amount of impurities can drastically decrease magnetization in the ground state. Introducing the picture of magnetic domain inversion, we have investigated impurity-density dependence of magnetization. In particular, we have shown that the magnetization with an infinitesimal density of impurities becomes as small as $57.7 \%$ of that without an impurity. The energy scale of this impurity effect is of the order of the remaining effective interaction between the spins adjacent to impurity sites. The low-energy excitations in doped systems are continuous from the lowest spin-state (except the finite-size gap). We also listed the conditions for this impurity effect.

In the materials which are effectively described by frustrated spin models, other interactions such as the Dzyaloshinsky-Moriya interaction or biquadratic interactions are sometimes not negligible. Although their influence on the impurity effect requires further study, the prediction in this paper deserves careful experimental investigations.

We thank M. Hase for valuable discussions and comments on related materials.
[1] T. Senthil, A. Vishwanath, L. Balents, S. Sachdev and M.P.A. Fisher, Science 303, 1490 (2004).

[2] N. Maeshima, K. Okunishi, K. Okamoto and T. Sakai, Phys. Rev. Lett. 93, 127203 (2004).

[3] D. Grohol, K. Matan, J.-H. Cho, S.-H. Lee, J. W. Lynn, D. G. Nocera and Y.S. Lee, Nature Mat. 4, 323 (2005).

[4] S. Fujiki and D.D. Betts, Can. J. Phys. 65, 76 (1987); Prog. Theor. Phys. Suppl. No. 87, 268 (1986).

[5] H. Nishimori and H. Nakanishi, J. Phys. Soc. Jpn. 57, 626 (1988).

[6] P.W. Leung and K.J. Runge, Phys. Rev. B 47, 5861 (1993).

[7] P.W. Anderson, Mater. Res. Bull. 8, 153 (1973).

[8] P. Fazekas and P. Anderson, Philos. Mag. 30, 432 (1974).

[9] H. Fukuyama, T. Tanimoto and M. Saito, J. Phys. Soc. Jpn. 65, 1182 (1996); H. Fukuyama, N. Nagaosa, M. Saito and T. Tanimoto, ibid. 65, 2377 (1996).

[10] M. Sigrist and A. Furusaki, J. Phys. Soc. Jpn. 652385 (1996).

[11] N. Nagaosa, A. Furusaki, M. Sigrist and H. Fukuyama, J. Phys. Soc. Jpn. 65, 3724 (1996).

[12] Y. Motome, N. Katoh, N. Furukawa and M. Imada, J. Phys. Soc. Jpn. 65, 1949 (1996).
[13] Y. Iino and M. Imada, J. Phys. Soc. Jpn. 65, 3728 (1996).

[14] M. Hase, K. Uchinokura, R.J. Birgeneau, K. Hirota and G. Shirane, J. Phys. Soc. Jpn. 65, 1392 (1996); M.C. Martin, M. Hase, K. Hirota, G. Shirane, Y. Sasago, N. Koide and K. Uchinokura, Phys. Rev. B 56, 3173 (1997).

[15] M. Azuma, Y. Fujishiro, M. Takano, M. Nohara and H. Takagi, Phys. Rev. B 55, R8658 (1997); M. Azuma, M. Takano and R.S. Eccleston, J. Phys. Soc. Jpn. 67, 740 (1998).

[16] T. Hamada, J. Kane, S. Nakagawa and Y. Natsume, J. Phys. Soc. Jpn. 57, 1891 (1988).

[17] S.R. White, Phys. Rev. Lett. 69, 2863 (1992); Phys. Rev. B 4810345 (1993).

[18] W. Marshall, Proc. Roy. Soc. A 232, 48 (1955).

[19] E.H. Lieb and D. Mattis, J. Math. Phys. 3, 749 (1962).

[20] In finite-size systems, there should be a gap due to the discreteness of energy levels. Here, we mean that an asymptotically continuous low-energy excitation exists which reflects the quantum nature of the system.

[21] For the model in Fig. [6] (a-2), the degrees of freedom of the spin on the decorating site connected to the impurity are neglected, since it behaves as a free spin. 